[0212-7199 (2006) 23: 7; pp 321-325] ANALES DE MEDICINA INTERNA Copyright (@) 2006 ARAN EDICIONES, S.L.

AN. MED. INTERNA (Madrid) Vol. 23, N. ${ }^{\circ} 7$, pp. 321-325, 2006

\title{
Tuberculosis en la comarca de Lorca: adaptarse o resistir
}

\author{
F. J. RUIZ LÓPEZ1,2, J. M. ZARAUZ GARCÍA ${ }^{1}$, M. M. ORTIZ ROMERO \\ J. R. VALERO MARTÍNEZ ${ }^{4}$, C. PEÑALVER MELLADO ${ }^{2}$, F. SÁNCHEZ GASCÓN ${ }^{2}$, \\ M. LORENZO CRUZ²
}

${ }^{1}$ Hospital Rafael Méndez. Lorca. ${ }^{2}$ Hospital Virgen de la Arrixaca. ${ }^{3}$ Hospital Reina Sofía. ${ }^{4}$ Consejería de Sanidad de la Región de Murcia. Murcia

TUBERCULOSIS IN THE AREA OF LORCA: TO ADAPT OR RESIST

\section{RESUMEN}

Objetivo: analizar las características epidemiológicas de los casos de tuberculosis del área de salud de la comarca de Lorca, su resistencia a fármacos y el impacto de la población inmigrante mayoritaria (Ecuador) en la misma

Pacientes y métodos: estudio longitudinal, retrospectivo de 6 años de duración (de enero de 1999 a diciembre 2004). Se recogieron datos epidemiológicos de cada caso, probabilidad diagnóstica inicial, tinciones diagnósticas, aislamientos, resistencia a fármacos, respuesta al tratamiento, así como, las diferencias epidemiológicas existentes entre inmigrantes y autóctonos.

Resultados: Se identificaron 158 casos, $41,7 \%$ eran inmigrantes con menos de 1 año de estancia en nuestro país, mayoritariamente procedentes de Ecuador. Observamos que la tuberculosis extrapulmonar para el clínico tenía una probabilidad diagnóstica baja, mientras que la pleural era alta, junto a síntomas como hemoptisis, dolor pleurítico y constitucionales. En 35,4\% de los casos se idéntifico germen por tinción en muestras respiratorias. Se obtuvo un $74 \%$ de resultados satisfactorios por el tratamiento y $13,9 \%$ de abandono asociado frecuentemente con la inmigración. La resistencia a isoniazida en no tratados fue del 5,3\% en el área, $9,5 \%$ en el caso de inmigrantes y 10,8\% en los oriúndos de Ecuador.

Conclusión: Se debe mejorar en: la detección de los casos extrapulmonares, evitar el abandono con la captación de los enfermos y la curación de enfermedad. Las resistencias a isoniazida en nuestra área sugiere adaptarse a tratamientos de inicio con cuatro fármacos y no a tres para evitar incremento de resistencias en nuestra área de salud.

PALABRAS CLAVE: Tuberculosis. Epidemiología. Inmigración. España. Ecuador. Resistencia.

\begin{abstract}
Objective: We studied the epidemiological characteristic of tuberculosis in Lorca area, drug resistant and the effect of the immigration population (Ecuador) in this area.

Patients and methods: A retrospective study for six years ago (1999, January to 2004, December) was realized. Epidemiological data, initial diagnostic probability, mycobacteria stain and cultive, drug resistance, treatment response, and epidemiological differences between immigrants and spain population were compared.

Results: Within 158 cases registred, 41.7 percent were immigrants with less than one year in Spain, the most part Ecuador population. We observed that extrapulmonar tuberculosis was lower initial diagnostic probability than pleural disease as well too haemoptisis, pleural pain and weight loss. It was identified positive micobacterial stain in 35.4 percent of respiratory samples. It was succesfully results in 74 percent of cases and 13.9 percent of patients were lost, this result was frecuently registred in immigrants, The isoniazide resistant in patients with not previous treatment was 5.3 percent and it was 9.5 percent in immigrants and 10.8 percent in Ecuador population.

Conclusion: It is necessary improve: the diagnostic of extrapulmonar tuberculosis and avoid loss patient by programs of capture and then the succesfully treatments raise. The isoniazide resistant in this area suggest that we must use initial four drug than tree and this manner not
\end{abstract} raise the drug resistant in the area.

KEY WORDS: Tuberculosis. Epidemiology. Immigration. Spain. Ecuador. Drug-resistant.

Ruiz López FJ, Zarauz, García JM, Ortiz Romero MM, Valero Martínez JR, Peñalver Mellado C, Sánchez Gascón F, Lorenzo Cruz M. Tuberculosis en la comarca de Lorca: adaptarse o resistir. An Med Interna (Madrid) 2006; 23: 321-325.

\section{INTRODUCCIÓN}

Es un hecho incuestionable, que la endemia de la enfermedad tuberculosa no solo afecta a países de baja renta per capita, también los llamados países desarrollados sufren la enfermedad sobre todo en las clases sociales más desfavorecidas. En nuestro país, se ha observado una disminución en la incidencia de casos, pero sin embargo países de nuestro entorno han incrementado su incidencia y más de la mitad de estos casos aparecen en inmigrantes (1). En determinadas áreas de España alcanzan hasta un $20 \%$ de los casos (2). Las comunicaciones favorecen la desaparición de fronteras y la población con bajo nivel socioeconómico emigra a otros países con más posibilidades de sustento o menos conflictos, modificando la incidencia de enfermedad en los países de acogida. La Región de Murcia esta muy influida por los flujos migratorios, basta

Trabajo aceptado: 24 de febrero de 2006 
con observar el padrón de habitantes para contemplar un crecimiento en la población inmigrante concretamente de América Latina y en el área de salud que nos ocupa, de nacionalidad ecuatoriana. Esta población procede de un país con alta incidencia de tuberculosis entre 25-49 casos/100.000 habitantes y estimada de 100 a 299 casos/100.000 habitantes (3) y que podría incrementar la incidencia de tuberculosis en el área de salud. Por otro lado, la propia Organización Mundial de la Salud (OMS) propone como metas de control global la curación de al menos el $85 \%$ de los enfermos bacilíferos (4). Sin embargo, en estudios previos de nuestra región se alcanzó el $60,2 \%$ de curaciones (5), por debajo de la OMS. Puesto que se desconocen algunos aspectos de la situación de la enfermedad en nuestra área, las características epidemiológicas de la población enferma, las resistencias a fármacos y el impacto de la población inmigrante más concretamente de origen ecuatoriana, nos planteamos este estudio revisando todos los casos acaecidos en un periodo de 6 años, para poder tomar decisiones tras los resultados.

\section{PACIENTES Y MÉTODOS}

Se realizó un estudio descriptivo, retrospectivo, longitudinal de 6 años de duración desde el 1 de enero de 1999 hasta el 31 diciembre del año 2004 del área III de Salud de la Región de Murcia, que comprende las localidades y diputaciones de Lorca, Totana, Puerto Lumbreras y Aledo, y que en el año 2004 comprende un total de 151352 habitantes (6).

En la identificación de los casos utilizamos cuatro fuentes de información: el registro de las enfermedades de declaración obligatoria del área de salud, el registro de aislamientos del laboratorio de microbiología del hospital del área Rafael Méndez de Lorca y por último, los registros procedentes de las historias clínicas del hospital y de atención primaria de salud.

En 1999 se acordó con el laboratorio de microbiología la realización de pruebas de sensibilidad a isoniazida, rifampicina, etambutol y estreptomicina en todos los aislamientos de micobacterias.

Los aislamientos se identificaron como complejo mycobacterium tuberculosis y solo en el caso de sospecha de mycobacterium bovis se realizó una identificación detallada de la especie.

Identificados los casos, se recogieron diferentes variables, como: año de aislamiento, sexo, edad, el país de origen del paciente, los antecedentes personales del mismo (factores de riesgo a VIH, patología renal, cardiaca, alcoholismo, adictos a drogas vía parenteral, EPOC, diabetes, neoplasias y silicosis); riesgo social (vagabundo, interno en prisión, residente de una institución cerrada, inmigrante reciente -menos de 1 año-, personal sanitario y contacto habitual de paciente con tuberculosis activa); tipo de tuberculosis (afectación de parénquima pulmonar, exclusivamente pleural, ganglionar, meníngea, genitourinaria, ósea y diseminada); antecedentes previos de tuberculosis; síntomas (asintomático, síntomas respiratorios inespecíficos -tos, sibilantes, disnea-, hemoptisis, dolor pleurítico, síntomas constitucionales, síntomas genitourinarios y otros); el patrón radiográfico de tórax (cavitación, infiltrado parenquimatoso, derrame pleural o normal); la probabilidad inicial diagnostica de la enfermedad para el médico (alta o media baja); el germen aislado; tinción positiva o negativa para bacilos ácido alcohol resistentes en la muestra remitida; resultados de cultivo Lowenstein; tratamiento recibidos (isoniazida (I) y rifampicina (R) 6-9 meses; I/R/ pirazinamida (P) 2 a 3 meses seguidos por I/R 3 a 4 meses; I/R/P/etambutol (E) o estreptomicina (Z) 2 a 3 meses seguido de I/R 3 a 4 meses; I/R/E 2 meses seguido por I/R 7 meses; I/R/P/E o Z seguido de I/R 7 meses, y otras pautas antibióticas; resistencia en no tratados a I, R, E, Z, resistencias en tratados previamente a I, $\mathrm{R}, \mathrm{E}, \mathrm{Z}$ y multirresistencia primaria (entendiendo la misma a I y R ) y multirresistencia adquirida; la respuesta al tratamiento: satisfactoria (curación), resultado parcialmente insatisfactorio (abandono del tratamiento, pérdida del paciente y fracaso del tratamiento) y exitus atribuible a la enfermedad, así como el tipo de muestra pedida para cada caso.

En el procedimiento estadístico se utilizó $\chi^{2}$ para valorar la asociación de variables cualitativas, aceptando $\mathrm{p}<0,05$ como significativo. Para valorar diferencias entre variables cuantitativas se utilizó el test no paramétrico de Wilcoxon, se tomo como significativo $\mathrm{p}<0,05$. Las variables cuantitativas se expresan como media \pm error estándar de la media.

\section{RESULTADOS}

Se consideró 158 casos de tuberculosis en el área de salud, con un pico de incidencia en los años 2000 y 2001 (Tabla I). El $65,8 \%(\mathrm{n}=104)$ de los casos eran varones y sin antecedentes de enfermedad crónica previa $(86,7 \%, \mathrm{n}=137)$, con edad media de $37,4 \pm 1,3$ años. Un alto porcentaje de los casos $(41,7 \%, \mathrm{n}=66)$ se detectaron en inmigrantes recientes, 6,9\% $(n=11)$ fueron contactos de enfermos con tuberculosis y no se encontró ningún riesgo social de enfermedad en 67 pacientes. La presentación de la enfermedad en buena parte de los casos fue pulmonar $(87,2 \%$, $\mathrm{n}=138)$; de los cuales el 14,5\% ( $\mathrm{n}=23)$ presentó derrame pleural. Los síntomas clínicos predominantes fueron cuadros compatibles con infección respiratoria $(67,7 \%, \mathrm{n}=107)$ y un $8,2 \%(\mathrm{n}=$ 13) de los pacientes presentaron hemoptisis.

\section{TABLA I}
EVOLUCIÓN ANUAL DE LA DECLARACIÓN OBLIGATORIA REALIZADA Y DE LOS CASOS CONSIDERADOS EN EL ESTUDIO ASÍ COMO LA INCIDENCIA DE CASOS EN EL AÑO CORRESPONDIENTE.

\begin{tabular}{lccc}
\hline Año & Declaración & Estudio & Incidencia/100.000 h \\
\hline 1999 & 14 & 15 & 11,55 \\
2000 & 38 & 36 & 27,46 \\
2001 & 31 & 36 & 27,46 \\
2002 & 29 & 23 & 17,54 \\
2003 & 23 & 24 & 17,10 \\
2004 & 28 & 24 & 16,42 \\
\hline
\end{tabular}

Más de un tercio de los casos fueron considerados inicialmente como baja probabilidad diagnostica $(37,3 \%, \mathrm{n}=58)$ y su diagnostico se indujo tras la identificación de microbiología o histología.

En este sentido, se encontró que la forma de presentación de la enfermedad se asociaba con la probabilidad diagnostica inicial, es alta en los casos de tuberculosis pleural y baja en los casos de localización extrapulmonar (Tabla II). También se observó asociación entre síntomas del paciente y sospecha inicial de diagnós- 
TABLA II

FORMA DE PRESENTACIÓN DE LA ENFERMEDAD

TUBERCULOSA Y SOSPECHA CLÍNICA A LA MISMA

\begin{tabular}{lcc}
\hline Presentación & $\begin{array}{c}\text { Baja probabilidad } n \\
(\%)\end{array}$ & $\begin{array}{c}\text { Alta probabilidad } n \\
(\%)\end{array}$ \\
\hline Extrapulmonar & $11(6,9 \%)^{*}$ & $8(5,1 \%)$ \\
Parenq. pulmonar & $40(25.3 \%)$ & $76(48,1 \%)$ \\
Pleural & $5(3,2 \%)$ & $18(11,4 \%)^{*}$ \\
Total & $56(35,4 \%)$ & $102(64,6 \%)$ \\
\hline
\end{tabular}

$\chi^{2}=6,12, p<0,05 .{ }^{*}$ Señala la asociación más frecuente.

tico, los síntomas genitorurinarios, enfermos asintomáticos y otros síntomas menores se asocian a diagnostico de baja probabilidad diagnóstica, mientras que hemoptisis y dolor pleurítico; y síntomas respiratorios se asocian a un diagnostico inicial de alta probabilidad clínica de tuberculosis (Fig. 1).

En la radiología de tórax aparecieron infiltrados en 37,5\% $(n=59)$ de los pacientes, cavitación en $32,4 \%(n=51)$ y derrame pleural en $14,5 \%(n=23)$. En $7,59 \%(n=12)$ de los pacientes no se realizó radiología de tórax siendo esta practica frecuente en presencia de tuberculosis genitourinaria $(6,33 \%$, $\left.\mathrm{n}=10 \mathrm{p}<0,0001, \chi^{2}=79,25\right)$.

En el 35,4\% $(n=56)$ de los casos, se identificó bacilos ácido alcohol resistentes en muestras respiratorias por tinción y en cultivo de las muestras respiratorias el 48,2\% $(n=92)$.

El diagnóstico se realizó por la respuesta a tratamiento de forma empírica en 26 casos (16,3\%). Doce de los cuales el cultivo para micobacterias resultó contaminado pero con tinción positiva. De los aislamientos sólo en 2 casos se identificó Mycobacterium bovis.

Respecto a las resistencias a fármacos encontramos un 5,3\% de enfermos no tratados previamente con resistencia a isoniazida y escasas resistencias en enfermos tratados (Tabla III).

Un 74\% $(\mathrm{n}=117)$ presentó un resultado satisfactorio al tratamiento, pero la población inmigrante se asoció a una mayor respuesta parcialmente insatisfactoria que los autóctonos, la mayoría por pérdidas $(\mathrm{n}=22(13,9 \%) v s . \mathrm{n}=8)($ Fig. 2$)$.

Con relación al tipo de tratamiento prescrito aproximadamente un tercio recibió 4 fármacos inicialmente y la mitad recibió tres fármacos (Fig. 3). Cuando analizamos las diferencias entre la población inmigrante y españoles, observamos que los inmigrantes son más jóvenes $(29,9 \pm 0,9$ vs. 44,3 $\pm 2,6$ años,

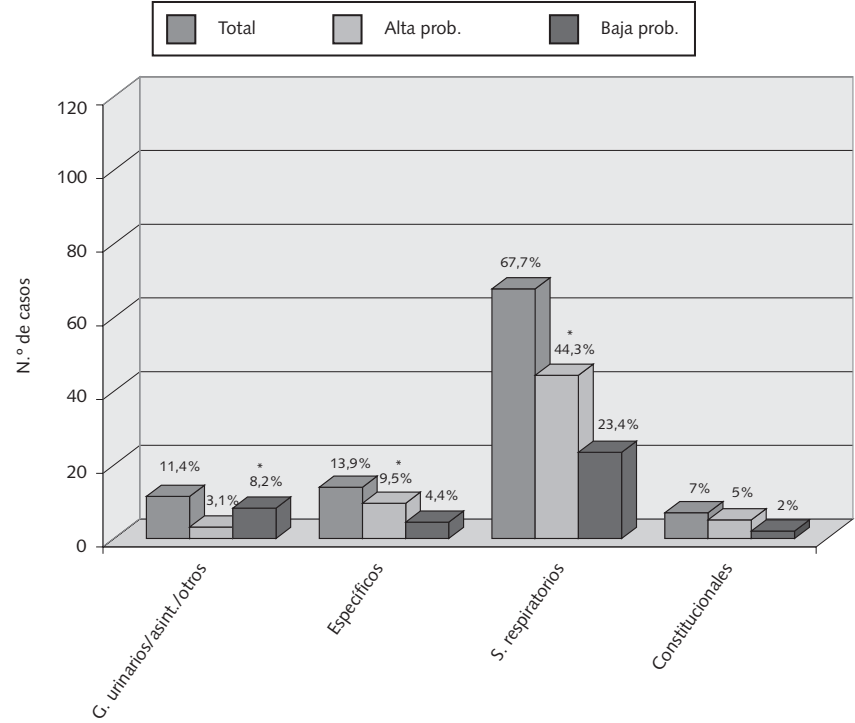

Fig. 1. Asociación entre síntomas del paciente agrupados (genitourinarios/asintomáticos/otros síntomas menores, específicos -hemoptisis y dolor pleurítico-, síntomas respiratorios comunes y sintomas constitucionales) y probabilidad diagnóstica para el médico, en total (barras blancas), alta probabilidad (barras rayadas), baja probabilidad (barras negras)* Asociación más frecuente $\left(\chi^{2}=10,37, p\right.$ $<0,05)$.

respectivamente $\mathrm{p}<0,001, \mathrm{~W} 4,52$ ), pero no existen diferencias en el sexo de los dos grupos. Tampoco se encontraron diferencias significativas en el tipo de presentación de la enfermedad, el riesgo social para la misma, la clínica, el patrón radiográfico y la sospecha clínica de enfermedad. Si que se observaron diferencias significativas respecto a las resistencias en pacientes no tratados a isoniazida, siendo esta más alta en inmigrantes (Tabla III), pero no existían diferencias en resistencias en tratados o primarias a rifampicina, etambutol y estreptomicina.

Particularmente en la población inmigrante procedente de Ecuador encontramos una alta resistencia en no tratados (Tabla III).

La población inmigrante recibió con más frecuencia 4 fármacos como pauta inicial de tratamiento mientras que los españoles recibieron 3 fármacos en los dos o tres primeros meses $\left(\mathrm{p}<0,0001, \chi^{2}=32,05\right)$.

TABLA III

DISTRIBUCIÓN DE RESISTENCIAS EN EL ESTUDIO EN NO TRATADOS PREVIAMENTE Y TRATADOS

\begin{tabular}{|c|c|c|c|c|c|c|}
\hline \multirow[b]{2}{*}{ Fármaco } & \multirow[b]{2}{*}{ Total n (\%) } & \multicolumn{2}{|c|}{ NO TRATADOS } & \multicolumn{2}{|c|}{ TRATADOS } & \multirow[b]{2}{*}{ Inmigrantes n (\%) } \\
\hline & & Inmigrantes n (\%) & Ecuatorianos n (\%) & Autóctonos n (\%) & Total n (\%) & \\
\hline Global & $9(6,8)$ & 7 & $6(13)$ & $2(2,4)$ & $1(0,7)$ & $1(0,76 \%)$ \\
\hline Isoniazida & $7(5,3)$ & $6(9,5)^{*}$ & $5(10,8)$ & $1(1,2)$ & $1(0,7)$ & $1(0,76 \%)$ \\
\hline Rifampicina & $1(0,7)$ & $1(1,6)$ & $1(2,1)$ & - & $1(0,7)$ & $1(0,76 \%)$ \\
\hline Etambutol & $2(1,5)$ & $1(1,6)$ & $1(2,1)$ & $1(1,2)$ & - & - \\
\hline Estreptomicina & $1(0,7)$ & - & - & $1(1,2)$ & - & - \\
\hline Combinada $(I+R)$ & $1(0,7)$ & $1(1,6)$ & $1(2,1)$ & - & & $1(0,76 \%)$ \\
\hline
\end{tabular}

Comparación de resistencias a fármacos entre población inmigrante y autóctona. $\chi^{2}=4,27, p<0,05 .{ }^{*}$ Indica frecuente asociación. Los porcentajes se refieren al total de cada subgrupo. No se encontraron resistencias en enfermos tratados para la población inmigrante ecuatoriana y autóctonos. 


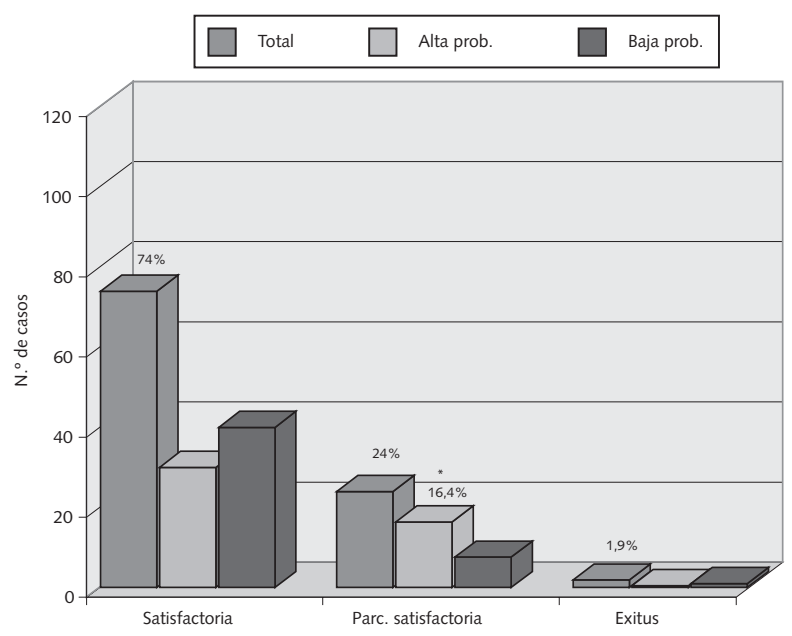

Fig. 2. Respuestas a tratamiento (satisfactoria, parcialmente satisfactoria y exitus) de la totalidad de casos, en pacientes inmigrantes (barra con trama) y nacionales (barra negra). *Asociación más frecuente $\left(\chi^{2}=7,13, p<001\right)$.

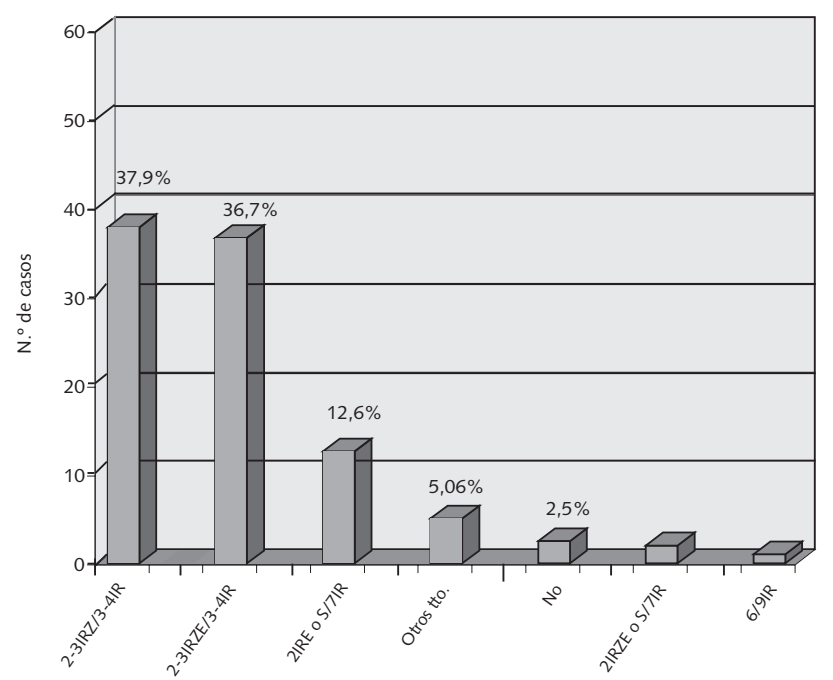

Fig. 3. Tratamientos prescritos a los enfermos del área. Isoniazida $(I)$, rifampicina $(R)$, etambutol $(E)$, pirazinamida $(Z)$, estreptomicina (S). Los números de cada categoría indican el número de meses de tratamiento..

\section{DISCUSIÓN}

La incidencia de tuberculosis en la comarca de Lorca ha estado históricamente por debajo de la media nacional. En los tres años previos al análisis se observó un progresivo descenso de la incidencia de 14,2 a 3,9 por 100.000 habitantes en los años 1996 y 1998 respectivamente $(7,8)$. Sin embargo, dicha tendencia cambió, y se alcanzó un pico en los años 2000 y 2001 para posteriormente disminuir en el año 2004 (por debajo de la media nacional). El número de casos declarados corresponde de forma aproximada a los analizados con variaciones anuales (según registro) excepto cinco casos que no cumplían las condiciones del estudio. En otras regiones espa- ñolas se observó una subnotificación cercana al 50\%, aunque en nuestro estudio, no se tuvo en cuenta el consumo de fármacos tuberculostáticos en el área de salud (9).

Buena parte del incremento en la incidencia de la enfermedad se debe a la detección de casos en la población inmigrante, con una mayor aportación que en otros estudios $(2,10)$. Más aún, la mayoría de los casos se presentan en inmigrantes con menos de 1 año de estancia en nuestro país, como en la comunidad autónoma de Madrid (10) y a diferencia de otros trabajos donde los casos de tuberculosis activa son más altas en inmigrantes que llevan más tiempo en España (11), posiblemente porque su situación es precaria por ingresos irregulares, hacinamiento etc. en su llegada a nuestro país. Estas diferencias sociales y sobre todo económicas, provocan la evolución a la enfermedad de infecciones latentes como es la tuberculosis (12). También influye en este incremento la incidencia de tuberculosis en Ecuador, mayor que la población autóctona (3).

Es llamativo la escasa influencia de otros factores de riesgo como coinfección con VIH, alcoholismo y adictos a drogas vía parenteral, confirmando la tendencia que apuntan otros autores (13).

En cuanto a la media de edad de 37 años y el predomino de casos en varones, los resultados son similares a otras zonas de España (14). La población inmigrante es bastante más joven que la autóctona como otros estudios (15).

El hecho de que una tercera parte de los casos tuvieran un diagnostico inicial de baja probabilidad de tuberculosis para el clínico, supone un retraso en el algoritmo diagnóstico del caso. En este sentido, encontramos que la presencia de tuberculosis extrapulmonar no es considerada inicialmente y más concretamente en presencia de síntomas genitourinarios. En este punto es inaceptable que determinados casos diagnosticados de tuberculosis genitourinaria no presentaban radiología de tórax. Mientras, la presencia de síntomas llamativos como la hemoptisis, el dolor pleurítico y los constitucionales se asocian a una alta probabilidad inicial de tuberculosis. Otros trabajos también recogen que la hemoptisis acorta el tiempo diagnostico además de la fiebre (16). La tuberculosis pleural se orientó a un diagnóstico de alta sospecha, probablemente por los datos bioquímicos del líquido. También, en otros estudios la presencia de tuberculosis extrapulmonar se diagnostica de forma tardía, mientras que la tuberculosis pleural de forma rápida, atribuible en este estudio a que los síntomas de dolor pleurítico son tan llamativos que provocan que el paciente acuda con mayor celeridad al sistema sanitario (17). Nuestro estudio no fue diseñado previamente para analizar la latencia en el diagnóstico pero sirve para llamar la atención sobre la tuberculosis extrapulmonar.

Si el factor decisivo en la aparición de nuevos casos es la presencia de bacilos en secreciones de vías aéreas inferiores, especialmente si se detectan por tinción, por su mayor concentración y sobre todo en los contactos de las personas que habitualmente conviven con el enfermo $(18,19)$. Se observó que la mitad de los pacientes presentaban bacilos en las muestras obtenidas de vías aéreas inferiores, y 35,4\% se visualizaban en la tinción, siendo esta cifra similar a otros estudios realizados en cataluña (20). Puesto que 1 caso infecta una media de 12 personas durante 1 año (21), es posible que en el área de salud estos 92 casos en 6 años supongan 1.004 individuos infectados o lo que es lo mismo una media de 167 casos infectados por año, de los cuales entre 5 a $15 \%$ desarrollaran la enfermedad a lo largo de su vida (22), en el mejor de los casos al menos 8 pacientes por año desarrollaran tuberculosis, la mitad aproximadamente de la 
media de casos nacionales en la actualidad, incidencia no modificable si no se consigue disminuir el número de casos o se realiza quimioprofilaxis adecuada a estos pacientes. Por ello es vital incentivar el estudio sistemático de contactos que habitualmente se realiza con los enfermos del área.

La resistencia a isoniazida en el área se sitúa por encima del $5 \%$, diferente de lo que ocurre en el conjunto de nuestro país o en áreas de salud cercanas a la nuestra. Estas zonas próximas presentan resistencias del 3\% (23) o como en el área de Cartagena en 1996, donde se hallaron resistencias a isoniazida del $4.17 \%$ (24) y siempre muy influenciada por la población inmigrante. Este dato nos induce a pensar la conveniencia de utilizar cuatro fármacos de inicio en nuestros casos de tuberculosis y no solo los enfermos inmigrantes como se suele utilizar en nuestra área a diferencia de lo recogido por otros autores (25), puesto que inmigrante o no, la convivencia habitual de la población puede reportar casos de resistencia en la población autóctona.

La mayoría de los casos de la población inmigrante son de nacionalidad ecuatoriana, como en otros trabajos (10) y el único estudio encontrado que evalúa resistencias en el país, revela un total de resistencias del $24 \%$ de los pacientes con un índice de resistencia a isoniazida del 14,2\%, a rifampicina $11,8 \%$ y multirresistencia de $8,9 \%$ en aquellos pacientes no tratados previamente (26), consuela que el porcentaje de resistencias de esta nacionalidad, en el área de salud, no tra- tados alcance solo 10,8\%, y no existe resistencia adquirida posiblemente porque la población que ya presentó tuberculosis en su país no inmigra, bien por que siguen enfermos o por las secuelas que presentan dado las dificultades de curación en el país de origen por su alta resistencias a fármacos. A la vista de nuestros resultados es de necesidad como se sugiere por otros autores (11) la realización de pruebas de sensibilidad a antibióticos sistemáticamente y la monitorización de las mismas anualmente dada la variabilidad potencial que provocan los flujos migratorios en las mismas y suprimir cualquier condicionante a su realización en las guías nacionales (27).

Por último, los resultados obtenidos con el tratamiento son similares a las nacionales, aunque muy por debajo de la propuesta de la OMS del 85\%. La mayor parte de los resultados potencialmente insatisfactorios se deben al abandono.

En conclusión, debemos mejorar en varios aspectos: en la detección de los casos extrapulmonares y su correcto manejo en pruebas complementarias, en programas de recaptación de los enfermos para evitar el abandono y mejorar la curación de enfermedad. La influencia de la población ecuatoriana en la tuberculosis de nuestra área es importante, nos obliga a adaptarnos a otras pautas de tratamiento con cuatro fármacos de inicio para de esta forma evitar incremento de resistencias en nuestra área de salud.

\section{Bibliografía}

1. Rieder HL. Epidemiology of tuberculosis in Europe. Eur Respir J, 1995; 20: 620S-632S

2. Grupo de trabajo de los talleres 2001 y 2002 de la unidad de investigación e tuberculosis de Barcelona. Prevención y control de las tuberculosis importadas. Med Clin (Barc) 2003; 121: 549-562.

3. WHO Report 2005. Global tuberculosis control, survellance, planning, finance. World Health Organization, 2005.

4. WHO Tuberculosis Program-Framework for effective tuberculosis control. World Health Organization. WHO/TB/94. 179.

5. Díez Ruiz-Navarro M, Hernández Espinosa JA, Caloto González T, Castells Carrillo C, Domínguez García A, García Fulgueiras AM et al. Resultados del tratamiento antituberculoso en seis comunidades autónomas españolas. Med Clin (Barc) 2001: 117: 474-480.

6. Padrón Municipal de Habitantes de la Región de Murcia. Disponible en http: //www.carm.es/econet/publica/padron.htm

7. Boletín epidemiológico de la Región de Murcia. Marzo 1997; 18(570).

8. Boletín epidemiológico de la Región de Murcia. Abril 1999; 20(595).

9. Criado-Álvarez JJ, Sanz Cortes J et al. El consumo de pirazinamida como indicador de la situación epidemiológica de la tuberculosis en Castilla- La Mancha. Rev Clin Esp 2004; 204: 298-302.

10. Arce Arnáez A, Iñigo Martínez J, Cabello Ballesteros L, Burgoa Arenales M. Tuberculosis e inmigración e un área sanitaria de Madrid. Situación epidemiológica y evolución en la década 1994-2003. Med Clin (Barc) 2005: 125: 210-212.

11. Gascón J. Enfermedades infecciosas e inmigración. Enferm Infecc Microbiol Clin, 2003 ; 21(10) : 535-539.

12. Jansá JM, García de Olalla P. Salud e inmigración: nuevas realidades y nuevos retos. Gac Sanit 2004; 18 (Supl.): 207-213.

13. Sánchez Gascón F Bernabeu Mora R. Inmigración y tuberculosis. Arch Bronconeumol 2003; 39: 5-7.

14. Toral Marín J, del Castillo Otero D, Carpio Muñoz V, González de Castro MA, Peñafiel Colas M. Unidad monográfica de tuberculosis: experiencia de 7 años de funcionamiento. Arch Bronconeumol 2000; 36: 191-196.

15. Ramos JM, Marín M, Rodríguez JC, Padilla I, Soler MJ, Gutiérrez F. Tuberculosis en inmigrantes: Diferencias clínicas y epidemiológicas cuando se comparan con la población nativa (1999-2002). Enferm Infecc Microbiol Clin 2004; 22: 315-318.
16. Altet Gómez MN, Alcaide Megías J, Canela Soler J, Milá Augé C, Jiménez Fuentes MA, de Souza Galvao ML et al. Estudio del retraso diagnóstico de la tuberculosis pulmonar sintomática. Arch Bronconeumol 2003; 39: 146-152.

17. Diez M, Bleda J, Alcaide T, Caloto C, Castells JI, Cardenal A, et al. Determinants of patient delay among tuberculosis care in Spain. European Journal of Public Health; Jun 2004; 14: 151-155.

18. Geus HAS, van Meijer J, Styblo K. Results of contac examination in Rotterdam, 1967-1969. Bull Int Union Tuber 1975; 50: 107-121.

19. Grzybowski S, Barnett GD, Styblo K. Contacts of cases of active pulmonary tuberculosis Bull Int Tuber 1975; 50: 90-106.

20. Godoy P, Dominguez J, Alcaide N, Camps N, Jansa S, Minguell JM. Characteristics of tuberculosis patients with positive sputum smear in Catalonia, Spain. European Journal of Public Health, 2004; 14: 71-75.

21. Slyblo K. Epidemiology of tuberculosis. The Hague, The Netherlands, Royal Netherlands Tuberculosis Association, 1991.

22. Walmsley SL. Screening and isoniazid prophylactic therapy for tuberculosis. En: Canadian Task Force on the Periodic Health Examination. Canadian Guide to Clinical Preventive Health Care. Ottawa: Health Canada, 1994; 754-65.

23. Marín Royo M, González Morán F, Moreno Muñoz R, Pardo Serrano F, Prada Alfaro P, Arnedo Pena A, et al. Evolución de las resistencias a Mycobacterium tuberculosis en la provincia de Castellón. Años 19921998. Arch Bronconeumol 200; 36: 551-556.

24. Rodríguez F, Garrido P, Nieto C. Estudio de Mycobacterium tuberculosis resistente a tuberculostáticos aislados en el Área de Salud de la Comunidad de Murcia (Cartagena). Boletín Epidemiológico de la Región de Murcia Julio 1996; 17(562).

25. Caminero JA. Origen, presente y futuro de las resistencias en tuberculosis. Arch Bronconeumol 2001; 37: 35-42.

26. Metz BZ, Douce RW, Brito N. Anti-tuberculosis drug resistance in two clinics in Ecuador. Int J Tuber Lung Dis 2000; 4: 115-117.

27. Alcaide Fernández de Vega F, Esteban Moreno J, González Martín J, Palacios Gutiérrez JJ. 9a. Micobacterias. 2005. En: Cercenado E y Cantón R. Procedimientos en Microbiología Clínica. Recomendaciones de la Sociedad Española de Enfermedades Infecciosas y Microbiología Clínica. Disponible en: http: //www.seimc.org/protocolos/microbiologia. 